\title{
Dihydrotanshinone I inhibits human glioma cell proliferation via the activation of ferroptosis
}

\author{
SHOUGANG TAN, XIAOQUN HOU and LIN MEI \\ Department of Neurosurgery, Qingdao Municipal Hospital, Qingdao, Shandong 266000, P.R. China
}

Received January 3, 2020; Accepted July 24, 2020

DOI: $10.3892 / \mathrm{ol} .2020 .11980$

\begin{abstract}
The aim of the present study was to investigate the effect of dihydrotanshinone I (DHI) on the survival of human glioma cells and the expression levels of ferroptosis-associated proteins. Human U251 and U87 glioma cells were cultured in vitro and treated with different concentrations of DHI and/or the ferroptosis inhibitor ferrostatin-1. A Cell Counting Kit- 8 assay was used to determine the cell survival rate. The cells were further analyzed to determine their 5-, 12- and 15-hydroxyeicosatetraenoic acid (HETE), lactate dehydrogenase (LDH) and malondialdehyde (MDA) levels, and reduced glutathione (GSH)/oxidized glutathione (GSSG) ratios. Western blotting was used to detect ferroptosis-associated glutathione peroxidase 4 (GPX4) and long-chain acyl-CoA synthetase 4 (ACSL-4). Changes in the mitochondrial membrane potential (MMP) were also observed using tetramethylrhodamine methyl ester staining and confocal fluorescence microscopy. The results revealed that DHI inhibited the proliferation of human glioma cells. Following treatment of the U251 and U87 cells with DHI, changes in the expression levels of ferroptosis-associated proteins were observed; the expression level of GPX4 decreased and that of ACSL-4 increased. DHI also increased the levels of LDH and MDA in the human glioma cells and reduced the GSH/GSSG ratio. The DHI-treated cells also exhibited a marked reduction in MMP. Furthermore, ferrostatin-1 blocked the DHI-induced effects in human glioma cells. From these results, it may be concluded that DHI inhibits the proliferation of human glioma cells via the induction of ferroptosis.
\end{abstract}

\section{Introduction}

Glioma is a type of brain tumor with very high recurrence and mortality rates (1). According to the American Cancer Society, $77 \%$ of patients with glioblastoma die within 1 year

Correspondence to: Professor Lin Mei, Department of Neurosurgery, Qingdao Municipal Hospital, 1 Jiaozhou Road, Qingdao, Shandong 266000, P.R. China

E-mail: 1inmei1@protonmail.com

Key words: dihydrotanshinone I, human glioma cells, ferroptosis of diagnosis (2). Gliomas are mainly caused by mutations in normal interstitial cells. The recurrence rate of glioma is positively associated with the degree of malignancy (3). With the continuous development of medical technology, the treatment of glioma is improving. Surgical resection continues to be the principal treatment for glioma, but is only applicable in patients with early-stage glioma $(4,5)$. The tumors of patients with early-stage gliomas are small, well-defined and easily removed surgically $(4,5)$. However, surgical resection is not an ideal treatment option for patients with malignant glioma because malignant gliomas are very large and present invasive growth (6). In recent years, the use of microscopy during surgery has enabled invasive gliomas to be resected more effectively (7). Radiation therapy is considered an important treatment option for various types of tumors, such as glioma, renal cell carcinoma and bladder cancer (8-10). A series of clinical studies have confirmed that radiotherapy can improve the survival rate of patients with glioma; however, radiotherapy is associated with numerous adverse reactions and side effects (11-13). Therefore, it remains important to identify potential drugs for the effective treatment of glioma.

15,16-Dihydrotanshinone I (DHI) is extracted from tanshen (Salvia miltiorrhiza bunge). Previous studies have shown that tanshen has various biological uses, which include the treatment of cardiovascular diseases, especially angina pectoris and myocardial infarction $(14,15)$. Lee and Lee (16) reported that DHI is able to inhibit the proliferation and induce the apoptosis of K562 leukemia cells. DHI has also been shown to inhibit breast and colon cancer through the mitochondrial apoptosis pathway $(17,18)$. Furthermore, DHI inhibits the proliferation of gastric cancer cells through the c-Jun N-terminal kinase/P38 signaling pathway (19). However, the effect of DHI on glioma cells and the underlying mechanisms have not yet been elucidated. The present study explored the antitumor effect and mechanism of DHI in gliomas.

Ferroptosis is a type of programmed cell death (20). The main morphological features of ferroptosis are shrinkage of the mitochondria and a reduction in the number of mitochondrial ridges (20). Ferroptosis of tumor cells, such as pancreatic and liver cancer cells, and normal tissue cells, including renal tubular cells and fibroblasts, can be induced by some small molecules and common clinical drugs, including vincristine, sorafenib and artemisinin (21-23). Ferroptosis is predominantly caused by the accumulation of intracellular lipid peroxides and the release of reactive oxygen species (ROS), which are 
closely associated with the accumulation of large amounts of iron ions in the cell. The iron chelator deferoxamine and the ferroptosis-specific inhibitors ferrostatin-1 or ferrostatin-1 are able to inhibit the ferroptosis process (24). The metabolism of iron ions and lipid peroxides is considered to be critical in the process of ferroptosis and to regulate its occurrence (25). Ferroptosis plays an important role in the development of tumors. The activation of ferroptosis has great therapeutic potential for tumors. Although the main regulatory network of ferroptosis has been established $(26,27)$, drugs that have been reported to regulate ferroptosis are limited in number and their exact regulatory mechanisms are unclear (28-30). Therefore, the identification of new drugs for ferroptosis regulation would be of great value.

A previous study indicated that tanshen induced ferroptosis in breast cancer cells, and significantly reduced the final tumor volume in a xenograft nude mouse model without adverse effects (31). Therefore, the present study aimed to evaluate the effects of DHI on the proliferation of glioma cells and investigate the contribution of ferroptosis to the underlying mechanism.

\section{Materials and methods}

Chemicals and treatment groups. DHI was obtained from ChemFaces Natural Products Co., Ltd. (cat. no. CFN-90162; purity, 98\%; solubility in DMSO, $>5 \mathrm{mg} / \mathrm{ml}$; PubChem CID: 11425923). HEB, U87 and U251 cells were treated with the DHI and/or the ferroptosis inhibitor ferrostatin-1, or with an equivalent volume of DMSO for $72 \mathrm{~h}$ at $37^{\circ} \mathrm{C}$ with $5 \%$ $\mathrm{CO}_{2}$. The cells were divided into four groups as follows: i) Control group, cells treated with DMSO; ii) DHI group, cells treated with DHI $(1,10,100$ or $1,000 \mu \mathrm{M})$; iii) ferroptosis inhibitor-control group, cells treated with ferrostatin-1 $(1 \mu \mathrm{M})$; iv) DHI + ferroptosis inhibitor group, cells treated with DHI $(100 \mu \mathrm{M})$ and ferrostatin-1 $(1 \mu \mathrm{M})$. Ferrostatin-1 was purchased from Santa Cruz Biotechnology, Inc.

Cell culture. The HEB human glial cell line was purchased from Shanghai Bioleaf Biotech Co., Ltd. The U251 human glioblastoma cell line was purchased from the European Collection of Authenticated Cell Cultures (cat. no. 09063001) and the U87 human glioblastoma cell line of unknown origin was purchased from American Type Culture Collection (ATCC; catalogue number: HTB-14). All the cell lines were identified by STR. All cells were cultured in DMEM (HyClone; GE Healthcare Lifesciences) supplemented with $10 \%$ fetal calf serum (Invitrogen; Thermo Fisher Scientific, Inc.) and penicillin-streptomycin combination $(100 \mathrm{mg} / \mathrm{ml}$; Thermo Fisher Scientific, Inc.), and maintained at $37^{\circ} \mathrm{C}$ in a $5 \% \mathrm{CO}_{2}$-humidified incubator.

Cell proliferation analysis. Cell proliferation was detected by Cell Counting Kit-8 (CCK-8) assay (cat. no. C0037; Beyotime Institute of Biotechnology). The HEB, U251 and U87 cells were inoculated on a 96-well plate at a density of 5,000 cells/well and treated as described above. Cell proliferation was detected at specified time points $(0,24$, 48 and 72 h) using a CellTiter $96{ }^{\circledR}$ AQueous One Solution Cell Proliferation Assay kit (Promega Corporation). The absorbance was measured at $450 \mathrm{~nm}$ using a microplate reader (Bio-Rad Laboratories, Inc.).

Western blot analysis. Total protein was extracted from HEB, U251 and U87 cells using the M-PER ${ }^{\mathrm{TM}}$ Mammalian Protein Extraction Reagent (Pierce; Thermo Fisher Scientific, Inc.). The protein concentration of the extract was determined using the BCA method. Equal amounts of protein extract and 2X SDS loading buffer were mixed and boiled for $5 \mathrm{~min}$. Proteins (30 $\mu \mathrm{g} /$ lane) were separated via SDS-PAGE on a $10 \%$ gel and then transferred to polyvinylidene difluoride membranes (Beijing Zhongshan Jinqiao Biotechnology Co., Ltd.). The membranes were blocked with $5 \%$ skimmed milk at room temperature for $2 \mathrm{~h}$ and then incubated with primary antibodies against glutathione peroxidase 4 (GPX4; 1:3,000; cat. no. sc-166570; Santa Cruz Biotechnology, Inc.), long-chain-fatty-acid-CoA ligase 4 (ACSL-4; 1:2,000; cat. no. sc-365230; Santa Cruz, USA) and anti- $\beta$-actin (1:500; cat. no. SA00001-9; ProteinTech Group Inc.) overnight at $4^{\circ} \mathrm{C}$. Following primary antibody incubation, the membranes were incubated with horseradish peroxidase-conjugated secondary antibodies $(1: 1,000$; cat. no. SA00001-9; ProteinTech Group, Inc.) at room temperature for $3 \mathrm{~h}$. Protein bands were observed using Amersham ECL Prime Western Blotting Detection Reagent (GE Healthcare Life Sciences) and scanned using Amersham Imager 600 (GE Healthcare).

5-Hydroxyeicosatetraenoic acid (HETE) assay. 5-HETE, a ferroptotic marker, was assessed using a 5-HETE ELISA kit (cat. no. CED739Ge; Wuhan USCN Business Co., Ltd.), according to the manufacturer's protocol.

12/15-HETE assay. The levels of 12- and 15-HETE, which are two ferroptotic markers, were determined using 12/15-HETE ELISA kits (cat. nos. ab133034 and ab133035; Abcam), according to the manufacturer's protocols.

Lactate dehydrogenase ( $\mathrm{LDH}$ ) assay. $\mathrm{LDH}$ is a cytoplasmic enzyme that is released from cells when they are damaged (32). LDH levels were measured using a colorimetric CytoTox 96 ${ }^{\mathrm{TM}}$ Cytotoxicity kit (cat. no. G1780; Promega Corporation) according to the manufacturer's protocol. Cells were treated with 10x lysis reagent to determine the maximum LDH release level. A 96-well plate reader (Molecular Devices, LLC) was used to measure the absorbance at $490 \mathrm{~nm}$. According to the manufacturer's instructions (33), the ratio of $\mathrm{LDH}$ release was calculated as the ratio of experimental $\mathrm{LDH}$ release to maximum LDH release.

Determination of the reduced glutathione (GSH)/oxidized glutathione (GSSG) ratio and malondialdehyde (MDA) levels. The levels of GSH and MDA in the cell extracts were investigated as described previously (34). The cellular extracts of the human glioma cell lines treated with DMSO, DHI $(100 \mu \mathrm{M})$ and/or ferrostatin-1 $(1 \mu \mathrm{M})$ at $37^{\circ} \mathrm{C}$ for $24 \mathrm{~h}$ were prepared according to the manufacturer's instructions. MDA levels were detected using a Lipid Peroxidation (MDA) assay kit (cat. no. K739-100; BioVision, Inc.) according to the manufacturer's instructions. The GSH/GSSG ratio was detected using 
A

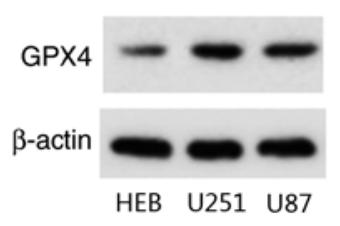

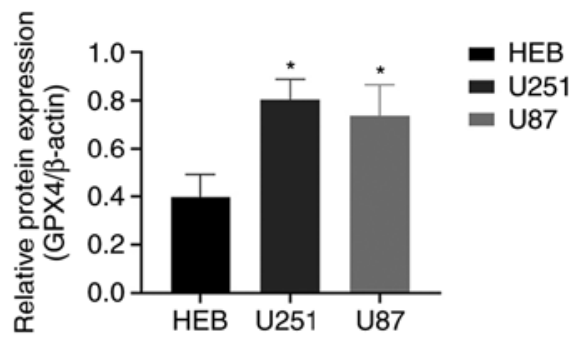

B
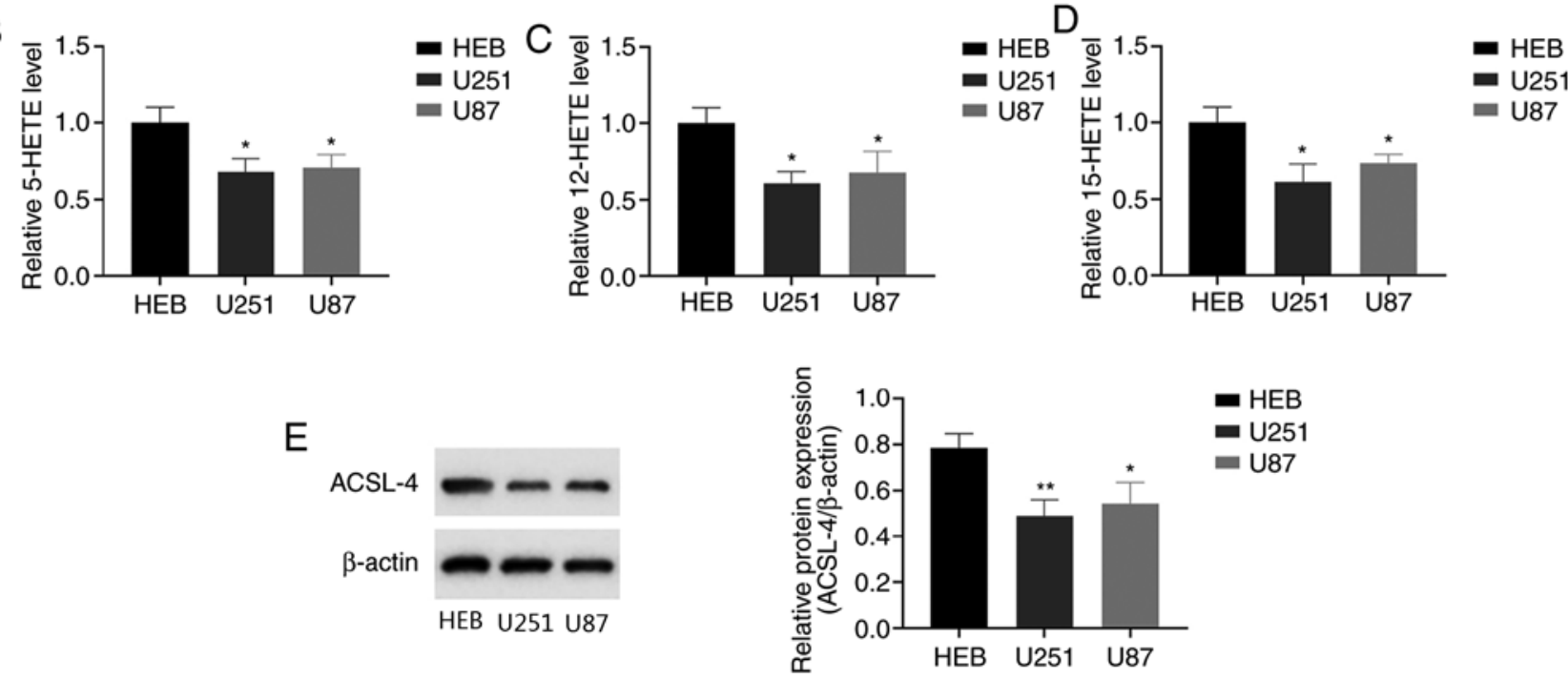

Figure 1. Indicators of ferroptosis in human glioma cells. (A) Representative western blots of GPX4 expression in human glioma and normal human glial cells, and quantification of the results. (B) 5-HETE levels, (C) 12-HETE levels and (D) 15-HETE levels detected in human and glioma cells normal glial cells using 5-, 12- and 15-HETE kits. (E) Representative western blots of ACSL-4 expression in normal human glioma cells, and quantification of the results. * $\mathrm{P}<0.05$ and ${ }^{* *} \mathrm{P}<0.01$ vs. HEB. GPX4, glutathione peroxidase 4; HETE, hydroxyeicosatetraenoic acid; ACSL-4, long-chain-fatty-acid-CoA ligase 4.

a Glutathione assay kit (cat. no. K264-100; BioVision, Inc.) according to the manufacturer's instructions.

Mitochondrial membrane potential (MMP) assay. The MMP of the cells was assayed using tetramethylrhodamine methyl ester (TMRM) according to the manufacturer's instructions (AAT Bioquest, Inc.). TMRM is readily sequestered by healthy mitochondria, but its fluorescence is rapidly lost when the MMP dissipates. In total, $1 \times 10^{6}$ cells were seeded in confocal dishes before treatment with DHI $(100 \mu \mathrm{M})$ with or without ferrostatin- $1(1 \mu \mathrm{M})$ at $37^{\circ} \mathrm{C}$ for $24 \mathrm{~h}$. The cells were washed twice with PBS, incubated for $20 \mathrm{~min}$ at $37^{\circ} \mathrm{C}$ with $100 \mathrm{nM}$ TMRM to stain the mitochondria and then carefully washed three times with PBS. Then, they were washed with PBS before nuclear staining with 4,6-diamidino-2-phenylindole (DAPI) (cat. no. 28718-90-3; Beyotime Institute of Biotechnology) for $15 \mathrm{~min}$ at room temperature. Finally, the cells were analyzed by confocal fluorescence microscopy (Nikon N-SIM; Nikon Corporation) to assess the intensity of red fluorescence (excitation at $549 \mathrm{~nm}$; emission at $573 \mathrm{~nm}$ ).

Statistical analysis. All data are presented as means \pm standard deviation. Each experiment was repeated three times. SPSS 22.0 software (IBM Corp.) was used for the statistical analysis. Two-tailed unpaired t-tests were used to compare two groups, while one- or two-way analysis of variance (ANOVA) was used to compare three or more groups. Pairwise group comparisons were conducted using Tukey's test as a post hoc test following ANOVA. $\mathrm{P}<0.05$ was considered to indicate a significant result.

\section{Results}

Ferroptosis levels are reduced in human glioma cells. Ferroptosis has been shown to be involved in the pathogenesis of gliomas (35). However, the relevant research is very limited. Therefore, in the present study, ferroptosis in human glioma cells was assessed by measuring the levels of GPX4, 5-, 12- and 15-HETE. GPX4 is an important membrane lipid peroxidase reductase, and the downregulation of GPX4 causes the accumulation of reactive oxygen species (ROS) on membrane lipids, which leads to ferroptosis (36). The latter three are lipid peroxidation products that are associated with the deposition of ferritin in ferroptosis, and recognized as indicators of ferroptosis levels $(20,26)$. Western blotting was performed to detect GPX4 expression in human glioma cells. The results revealed that GPX4 levels were significantly higher in human glioma cells compared with normal human glial cells (Fig. 1A). ELISAs were performed to evaluate the levels of 5-, 12- and 15-HETE in the cells. The 5-, 12- and 15-HETE levels were significantly decreased in the glioma cells compared with the control glial cells (Fig. 1B-D). These results demonstrate that U251 and U87 human glioma cells have higher levels of GPX4 and lower 5-, 12- and 15-HETE levels than normal human glial cells.

ACSL-4 is considered to be a key regulatory gene for ferroptosis. Previous studies have shown that ACSL-4 
A

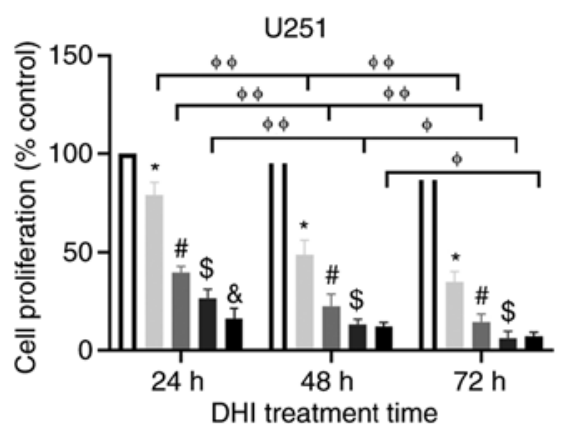

C

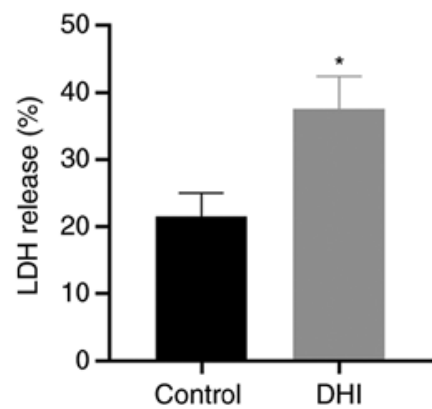

$\mathrm{B}$
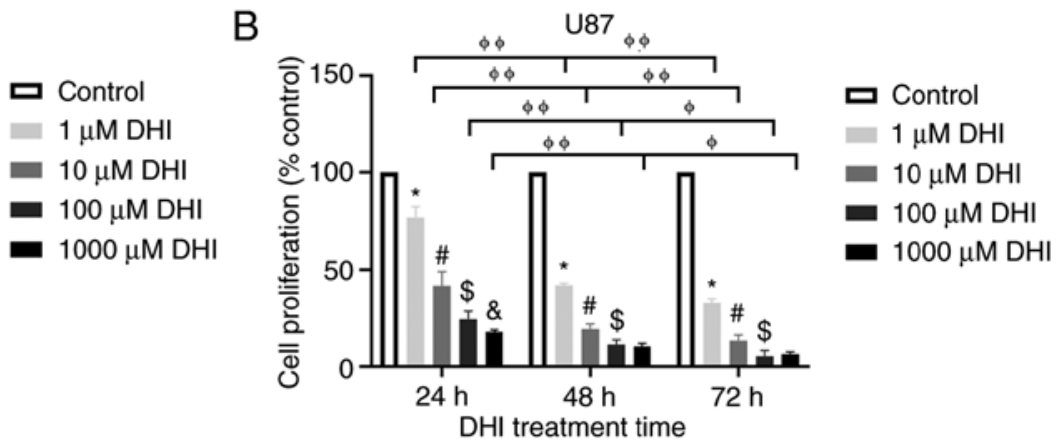

D

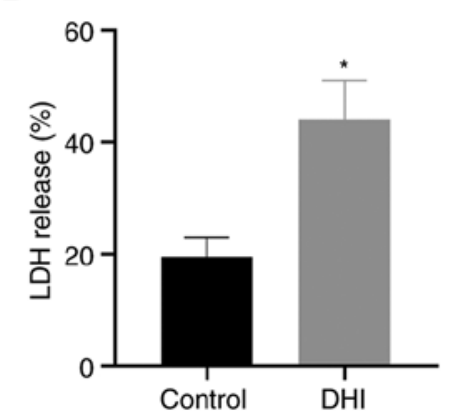

Figure 2. DHI blocks the proliferation of human glioma cells. The proliferation of (A) U251 and (B) U87 cells was measured using Cell Counting Kit-8 assay. The LDH levels of (C) U251 and (D) U87 cells. " $\mathrm{P}<0.01$ vs. control; " $\mathrm{P}<0.01$ vs. DHI $(1 \mu \mathrm{M}) ;{ }^{\$} \mathrm{P}<0.05$ vs. DHI $(10 \mu \mathrm{M}) ;{ }^{\&} \mathrm{P}<0.05$ vs. DHI $(100 \mu \mathrm{M}) ;{ }^{\Phi \Phi} \mathrm{P}<0.01$ and ${ }^{\Phi} \mathrm{P}<0.05$ as indicated. DHI, 15,16-dihydrotanshinone I; LDH, lactate dehydrogenase.

overexpression upregulates ferroptosis levels in gliomas (37). Compared with normal human glial cells, the human glioma cells exhibited significantly lower ACSL-4 levels (Fig. 1E). The reduction in ACSL-4 expression may be associated with dysfunctional ferroptosis in glioma. These results indicate that ferroptosis levels are reduced in glioma cells.

DHI inhibits the proliferation of human glioma cells. To investigate the effect of DHI on glioma cells, two glioma cell lines were treated with different concentrations of DHI for 24, 48 and $72 \mathrm{~h}$. The CCK8 assay was used to measure cell proliferation. The cell viability of the U251 and U87 glioma cell lines was significantly reduced after treatment with DHI (Fig. 2A and B). Fig. 2A shows that DHI had significant time- and dose-dependent effects on U251 glioma cells. The inhibitory effect on cell growth increased as the treatment time increased. At each time point, the inhibition rates of higher concentrations of DHI were higher than those of lower concentrations of DHI in the majority of cases. However, no significant difference was observed between the antiproliferative effects of 100 and $1,000 \mu \mathrm{M}$ DHI following treatment for either 48 or $72 \mathrm{~h}$. Therefore, DHI at a concentration of $100 \mu \mathrm{M}$ was used for subsequent experiments. When the experiment was repeated using U87 cells, similar results to those in U251 cells were obtained (Fig. 2B).

To test whether DHI affects the cytotoxicity of human glioma cells, the levels of LDH in the cells were examined. The more LDH is released, the more severe the cell damage, which results in a reduction in cell proliferation. Following DHI treatment, the release of LDH increased significantly (Fig. 2C and D). These data indicate that DHI reduces the viability of human glioma cells.
DHI induces ferroptosis in human glioma cells by downregulating GPX4 and upregulating ACSL-4. To evaluate whether the inhibition of cell proliferation by DHI is associated with ferroptosis, the mechanism of action of DHI in human glioma cells was investigated. Lipid peroxidation is a key process during ferroptosis (38). MDA is a natural product of lipid peroxidation that is often used as a marker for lipid peroxidation. The results of the present study indicate that following treatment with $100 \mu \mathrm{M}$ DHI for $24 \mathrm{~h}$, the MDA levels of the U251 and U87 cells were significantly increased (Fig. 3A and B).

GSH and GSSG constitute an important cellular antioxidant system that provides a reducing environment for the reduction of oxidative substances. GPX4 is an important regulator of ferroptosis, the absence of which causes a sharp increase in GSSG, leading to a reduction in the GSH/GSSG ratio (39) GSH and GSSG constitute a critical cellular antioxidant system and provide a reducing environment to reduce oxidative species. The loss of GPX4 activity can cause a drastic increase in GSSG, leading to a decrease in the GSH/GSSG ratio and ultimately ferroptosis (30). In the present study, it was found that after $24 \mathrm{~h}$ of DHI treatment, intracellular GPX4 expression in human glioma cells was significantly inhibited (Fig. 3C and D). Notably, DHI also caused significant reductions in GSH levels and the GSH/GSSG ratio (Fig. 3E-H). In addition, DHI significantly upregulated ACSL-4 expression in human glioma cells (Fig. 3C and D). These results indicate that ferroptosis is one of the mechanisms by which DHI induces cell death in human glioma cells.

DHI induces a reduction in the MMP of human glioma cells. The probe TMRM was used to detect the MMP of the glioma 
A

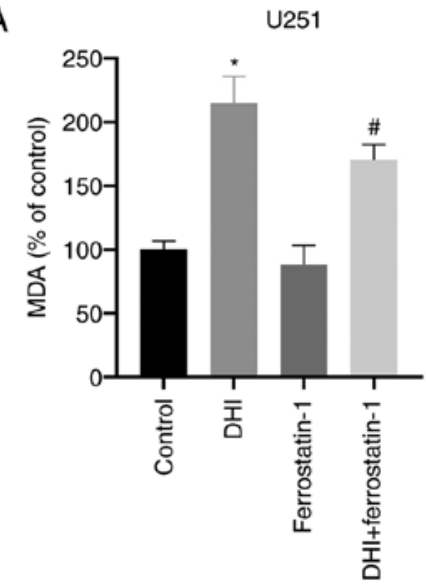

B

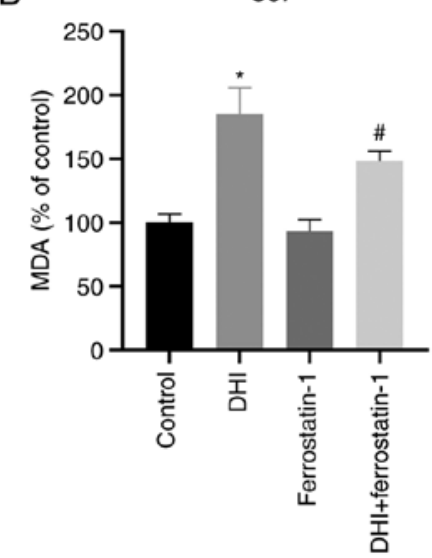

C
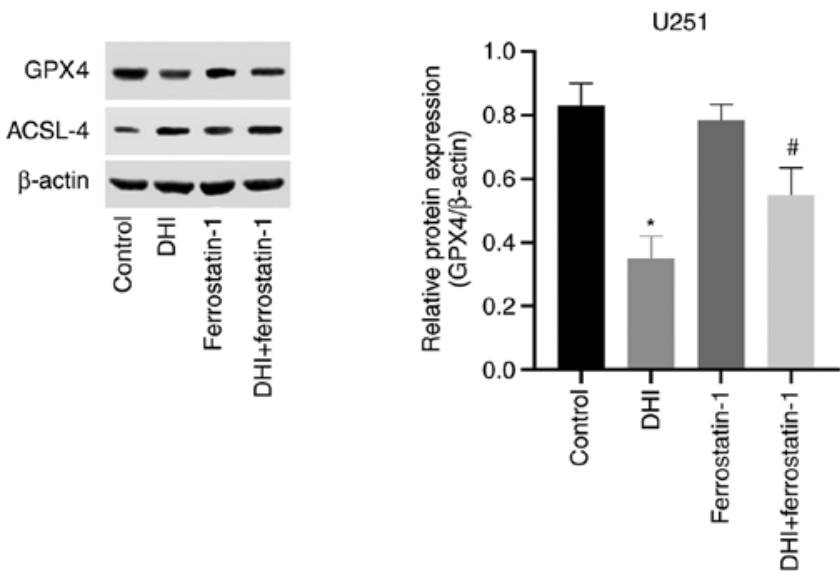

U87

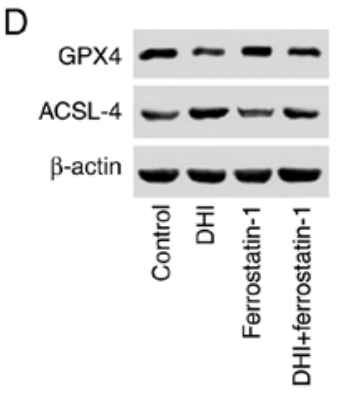



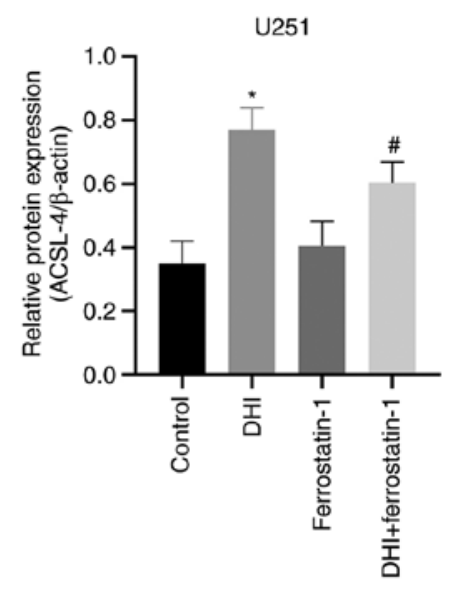

U87

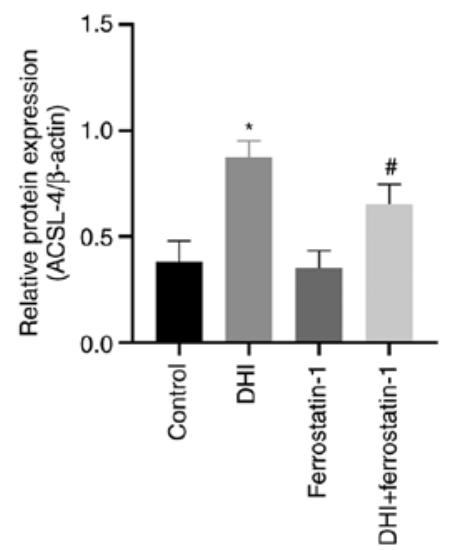

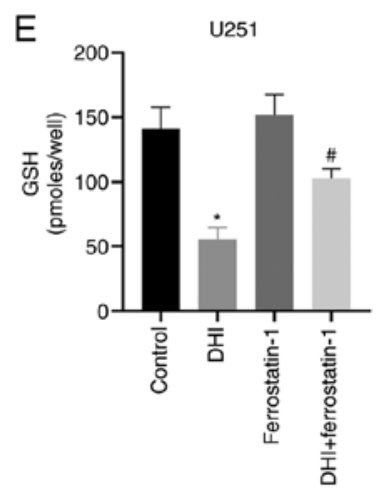
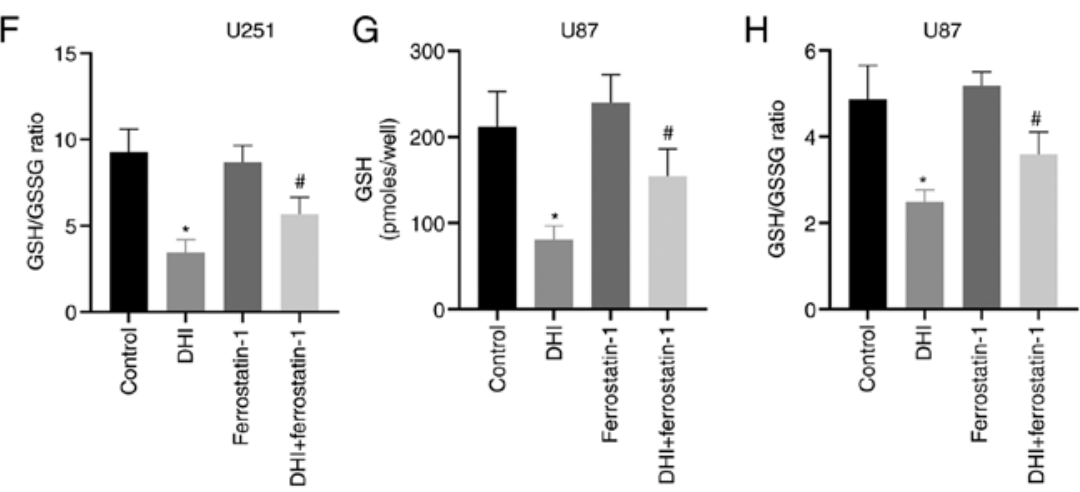

Figure 3. DHI inhibits the proliferation of glioma cells via the regulation of ferroptosis. U251 cells and U87 cells were treated with DHI (100 $\mu \mathrm{M})$ and/or the ferroptosis inhibitor ferrostatin-1 ( $1 \mu \mathrm{M})$. The MDA levels of (A) U251 and (B) U87 cells. Representative western blots of GPX4 and ACSL-4 expression in (C) U251 and (D) U87 human glioma cells, and quantification of the results. (E) The GSH levels and (F) GSH/GSSG ratios of U251 cells and (G) the GSH levels and (H) GSH/GSSG ratios U87 cells. "P<0.01 vs. control group; ${ }^{*} \mathrm{P}<0.05$ vs. DHI. DHI, 15,16-dihydrotanshinone I; MDA, malondialdehyde; GPX4, glutathione peroxidase 4; ACLS-4, long-chain-fatty-acid-CoA ligase 4; GSH, reduced glutathione; GSSG, oxidized glutathione. 
A
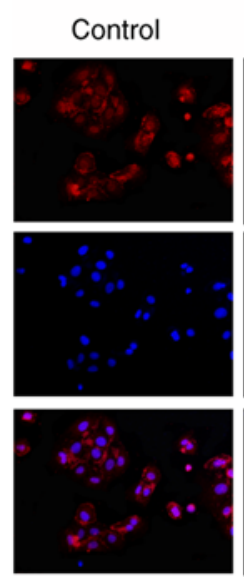

U251
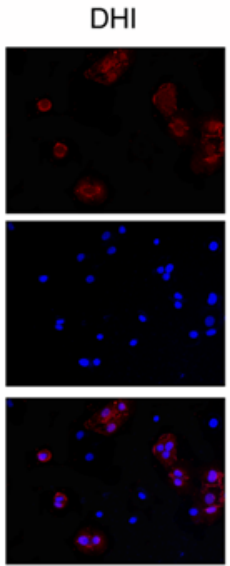

DHI+ferrostatin-1
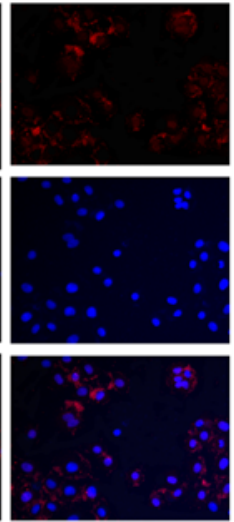

B

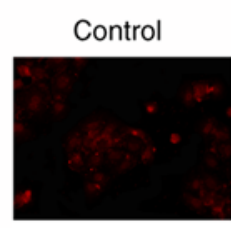

DAPI

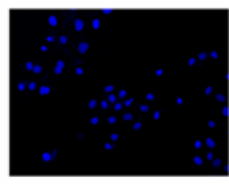

Merge

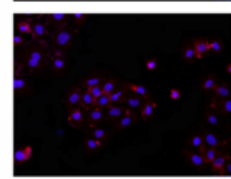

U87


Figure 4. DHI reduces in the mitochondrial membrane potential of human glioma cells. The mitochondrial membrane potential of (A) and (B) U87 cells following TMRM staining observed using confocal fluorescence microscopy (magnification, x400). DHI, 15,16-dihydrotanshinone I; TMRM, tetramethylrhodamine methyl ester.

A

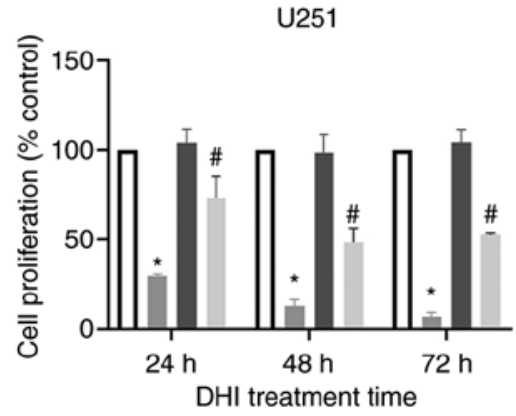

B

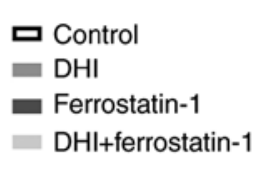

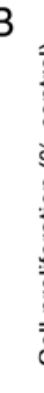

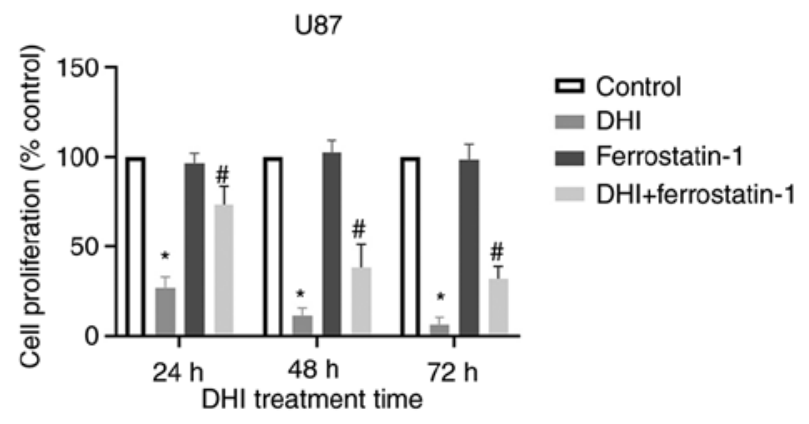

Figure 5. Ferrostatin-1 blocks the inhibitory effect of DHI on human glioma cells. The proliferation of (A) U251 and (B) U87 cells was measured using Cell Counting Kit- 8 assay. ${ }^{*} \mathrm{P}<0.01$ vs. control group; ${ }^{*} \mathrm{P}<0.01$ vs. DHI. DHI, 15,16 -dihydrotanshinone I.

cells. TMRM accumulates in the mitochondria of living cells and produces bright red fluorescence. DAPI makes cells produce blue fluorescence in order to locate cells. Confocal microscopy of TMRM-stained cells revealed that glioma cells treated with DHI showed reduced red fluorescence compared with that of untreated cells. These results indicate that DHI induces a reduction in the MMP of glioma cells (Fig. 4).

Anticancer effect of DHI on human glioma cells can be blocked by ferroptosis inhibitors. Ferrostatin-1 is a ferroptosis inhibitor that reduces the accumulation of intracellular ROS and cell death. A previous study demonstrated that ferrostatin-1 is able to inhibit the induction of ferroptosis in vitro (40). The potent inhibition of ferroptosis by ferrostatin-1 has been ascribed to its ability to slow the accumulation of lipid hydroperoxides (41). Ferrostatin-1 was used to determine whether DHI can induce ferroptosis in human glioma cells in the present study. The western blotting results indicate that ferrostatin-1 inhibited the DHI-induced reductions in GPX4 expression levels and increases in ACSL-4 expression levels in U251 and U87 cells (Fig. 3C and D). Furthermore, ferrostatin-1 attenuated the increases in MDA levels (Fig. 3A and B) and reductions in GSH levels and GSH/GSSG ratio that were induced by DHI (Fig. 3E-H). Also, confocal microscopy of TMRM-stained cells revealed that glioma cells treated with DHI and ferrostatin-1 exhibited increased red fluorescence compared with that of glioma cells treated with DHI alone.

The effect of ferrostatin-1 on the proliferation of human glioma cells was evaluated using the CCK- 8 assay. The results revealed that ferrostatin-1 increased the proliferative activity of DHI-treated human glioma cells compared with that of cells treated with DHI alone (Fig. 5). Therefore, it may be concluded that ferrostatin-1 blocks the inhibitory effect of DHI on human glioma cell proliferation to a certain extent.

\section{Discussion}

Increasing attention is being paid to the antitumor activity of natural substances, a number of which are being developed into antitumor drugs. Tanshen is one of the most commonly used natural medicines in clinical practice. It has been reported to have cardiovascular effects, including increased coronary blood flow, the expansion of coronary arteries, and prevention of myocardial infarction and myocardial ischemia, as well as being used to treat gastric cancer, liver cancer, leukemia and cervical cancer $(42,43)$. Tanshinones are the main components of tanshen, and tanshinone IIA is one of the most abundant tanshinones. Therefore, previous research has 
focused on the antitumor activity of tanshinone IIA $(44,45)$. However, there are few studies on other single agents that may be isolated from tanshen, including DHI, tanshinone I and cryptotanshinone (CPT). Liu et al (46) explored the inhibitory effect of tanshinones on lung cancer cells, and Mosaddik (47) investigated the inhibitory effects of tanshinones on leukemia cells. These studies demonstrated that the inhibitory effect of DHI on tumor cells was stronger than that of tanshinone IIA, tanshinone I and CPT. DHI is easy to extract, has low toxicity against normal cells and is a promising natural antitumor drug (48). A number of studies have reported the antitumor mechanism of DHI (49-51). A previous study revealed that DHI exerts antitumor effects by inhibiting the proliferation of cancer cells, inducing cancer cell apoptosis and promoting differentiation (52). However, there are relatively few studies on the effect of DHI on glioma.

Gliomas are the malignant tumors with the highest incidence in the central nervous system in adults (53). Due to substantial heterogeneity and invasive growth characteristics, glioma is very difficult to cure completely (54). As research has advanced, new therapies such as immunotherapy and gene therapy have been developed but the survival time of glioma patients has not been significantly prolonged $(55,56)$. Therefore, it is necessary to find new and effective drugs for the targeted treatment of gliomas. The main aim of the present study was to explore the mechanism by which DHI inhibits human glioma cell proliferation and promotes human glioma cell death.

In the present study, the human glioma cell lines U251 and U87 were used to investigate the effect of DHI on the proliferation of human glioma cells and its mechanism. The results of the CCK8 assay showed that the proliferation of human glioma cells was significantly inhibited following treatment with DHI for 24,48 and $78 \mathrm{~h}$; the antiproliferative effect was time- and concentration-dependent

Ferroptosis is a mode of iron-dependent cell-regulated death. It is characterized by the abnormal accumulation of iron-dependent lipid peroxides (lipid ROS) in cells, which disrupts the redox balance and ultimately leads to cell death (57). These processes are caused by the loss of intracellular GPX4 activity. The cell membrane-localized system $\mathrm{Xc}$ - is composed of SLC3A2 and SLC7A11 (58). SLC3A2 is a binding protein of SLC7A11, which takes up cystine and excretes glutamate (58). In cell metabolism, GSH is synthesized from cystine and is an important antioxidant in the cell (59). It scavenges free radicals and maintains the redox balance inside and outside the cell (59). SLC7A11 is highly expressed in a variety of tumors; by increasing cystine uptake it leads to increased intracellular GPX4 synthesis, reduced intracellular oxidative stress and the suppression of ferroptosis, thereby promoting tumor growth $(60,61)$. GPX4 is a GSH-dependent enzyme, and selenocysteine, which is one of the amino acids in the active center of GPX4, can be inserted into GPX4 through selenocysteine transfer RNA (tRNA) $(62,63)$. In addition, the maturation of selenocysteine tRNA may also be regulated by the mevalonate pathway to act on GPX4 $(62,63)$. GSH and selenocysteine both regulate the occurrence of ferroptosis in cells by affecting GPX4. GPX4 has been recognized as a key target in the induction of ferroptosis by various agents, including erastin and RSL3 (64). Erastin consumes GSH to inhibit the activity of GPX4, while RSL3 directly inhibits the activity of GPX4 (64). Therefore, the reduction of GPX4 activity appears to be a necessary condition for ferroptosis. The role of ferroptosis in tumorigenesis is becoming a prominent topic of research, and a number of studies have shown that multiple drugs are able to induce ferroptosis in tumor cells and affect the expression of ferroptosis-associated genes (65-67). Previous studies have found that cisplatin induces ferroptosis in A549 and HCT116 cells and cause changes in GPX4 expression (68). Similarly, sorafenib targets the Xc-system and causes ferroptosis in various cancer cells such as liver cancer cells $(69,70)$. In addition, sulfasalazine induces ferroptosis in breast cancer cells, and the mechanism may be associated with the increased expression of DMT1 Mrna (71). Furthermore, a number of studies have shown that fatty acid metabolism is essential for maintaining the microenvironment of malignant tumors and participating in the occurrence and development of tumors (72,73). ACSL4 serves a key role in fatty acid metabolism. Previous studies have shown that ACSL4 activates long-chain unsaturated fatty acids to participate in the synthesis of membrane phospholipids and trigger cell ferroptosis (74). Ferroptosis is associated with tumor suppression and is a potential therapeutic mechanism for the treatment of tumors. ACSL4 may be used as an indicator to predict whether cells have the potential to undergo ferroptosis (37). The present study confirmed that DHI affects the expression of ferroptosis-related genes in human glioma cells. After DHI treatment, the expression of GPX4 in human glioma cells decreased, while the expression of ACSL-4 increased. However, following treatment with the ferroptosis inhibitor ferrostatin-1, the expression of GPX4 in human glioma cells increased, the expression of ACSL-4 decreased, and the cell survival rate increased significantly. These results indicate that the effect of DHI on human glioma cells was mediated by ferroptosis to a certain extent.

In summary, the results of the present study confirm that DHI attenuates the proliferation of human glioma cells by inducing ferroptosis. They also provide a greater understanding of the mechanism of DHI that may be of benefit in the development of methods using DHI for the treatment of glioma.

\section{Acknowledgements}

Not applicable.

\section{Funding}

No funding was received.

\section{Availability of data and materials}

The datasets used and/or analyzed during the current study are available from the corresponding author on reasonable request.

\section{Authors' contributions}

LM conceived and designed the study. ST performed the experiments. ST and XH analyzed the data and wrote the manuscript. All authors read and approved the final manuscript and agreed to be accountable for all aspects of the research. 


\section{Ethics approval and consent to participate}

Not applicable.

\section{Patient consent for publication}

Not applicable.

\section{Competing interests}

The authors declare that they have no competing interests.

\section{References}

1. Ohtaki S, Wanibuchi M, Kataoka-Sasaki Y, Sasaki M, Oka S, Noshiro S, Akiyama Y, Mikami T, Mikuni N, Kocsis JD and Honmou O: ACTC1 as an invasion and prognosis marker in glioma. J Neurosurg 126: 467-475, 2017.

2. Ohgaki H and Kleihues P: Population-based studies on incidence, survival rates, and genetic alterations in astrocytic and oligodendroglial gliomas. J Neuropathol Exp Neurol 64: 479-489, 2005.

3. Hadjipanayis CG and Van Meir EG: Tumor initiating cells in malignant gliomas: Biology and implications for therapy. J Mol Med (Berl) 87: 363-374, 2009.

4. Becker D, Scherer M, Neher P, Jungk C, Jesser J, Pflüger I, Brinster R, Bendszus $\mathrm{M}$, Bruckner $\mathrm{T}$, Maier-Hein $\mathrm{K}$ and Unterberg A: Going beyond Diffusion Tensor Imaging tractography in eloquent glioma surgery-high resolution fiber tractography: Q-ball or constrained spherical deconvolution? World Neurosurg 134: e596-e609, 2020.

5. Cajigas I, Mahavadi AK, Shah AH, Borowy V, Abitbol N, Ivan ME, Komotar RJ and Epstein RH: Analysis of intra-operative variables as predictors of 30-day readmission in patients undergoing glioma surgery at a single center. J Neurooncol 145 : 509-518, 2019

6. Slof J, Diez Valle R and Galvan J: Cost-effectiveness of 5-aminolevulinic acid-induced fluorescence in malignant glioma surgery. Neurologia 30: 163-168, 2015 (In En, Spanish).

7. Brokinkel B, Yavuz M, Warneke N, Brentrup A, Hess K, Bleimüller C, Wölfer J and Stummer W: Endoscopic management of a low-grade thalamic glioma: A safe alternative to open microsurgery? Acta Neurochir (Wien) 159: 1237-1240, 2017.

8. Maruyama A, Yasuoka S, Katoh N and Asai J: Radiation-induced osteosarcoma of the skull mimicking cutaneous tumor after treatment for frontal glioma. J Dermatol 47: 69-71, 2020.

9. Siva S, Kothari G, Muacevic A, Louie AV, Slotman BJ, The BS and Lo SS: Radiotherapy for renal cell carcinoma: Renaissance of an overlooked approach. Nat Rev Urol 14: 549-563, 2017.

10. Milosevic M, Gospodarowicz M, Zietman A, Abbas F, Haustermans K, Moonen L, Rödel C, Schoenberg M and Shipley W: Radiotherapy for bladder cancer. Urology 69 (1 Suppl): S80-S92, 2007.

11. Bitterman DS, MacDonald SM, Yock TI, Tarbell NJ, Wright KD, Chi SN, Marcus KJ and Haas-Kogan DA: Revisiting the role of radiation therapy for pediatric low-grade glioma. J Clin Oncol 37: 3335-3339, 2019

12. Lu VM, Welby JP, Laack NN, Mahajan A and Daniels DJ Pseudoprogression after radiation therapies for low grade glioma in children and adults: A systematic review and meta-analysis. Radiother Oncol 142: 36-42, 2020

13. Kieran MW, Goumnerova L, Manley P, Chi SN, Marcus KJ, Manzanera AG, Silva Polanco ML, Guzik BW, Aguilar-Cordova E, Diaz-Montero CM, et al: Phase I study of gene-mediated cytotoxic immunotherapy with AdV-tk as adjuvant to surgery and radiation for pediatric malignant glioma and recurrent ependymoma. Neuro Oncol 21: 537-546, 2019.

14. Wang X, Morris-Natschke SL and Lee KH: New developments in the chemistry and biology of the bioactive constituents of Tanshen. Med Res Rev 27: 133-148, 2007.

15. Tzen JT, Jinn TR, Chen YC, Li FY, Cheng FC, Shi LS, She HK, Chen BC, Hsieh V and Tu ML: Magnesium lithospermate B possesses inhibitory activity on $\mathrm{Na}+\mathrm{K}+-\mathrm{ATPase}$ and neuroprotective effects against ischemic stroke. Acta Pharmacol Sin 28: 609-615, 2007.
16. Lee DS and Lee SH: Biological activity of dihydrotanshinone I: Effect on apoptosis. J Biosci Bioeng 89: 292-293, 2000.

17. Tsai SL, Suk FM, Wang CI, Liu DZ, Hou WC, Lin PJ, Hung LF and Liang YC: Anti-tumor potential of 15,16-dihydrotanshinone I against breast adenocarcinoma through inducing $\mathrm{G} 1$ arrest and apoptosis. Biochem Pharmacol 74: 1575-1586, 2007.

18. Wang L, Hu T, Shen J, Zhang L, Chan RLY, Lu L, Li M, Cho CH and Wu WKK: Dihydrotanshinone I induced apoptosis and autophagy through caspase dependent pathway in colon cancer. Phytomedicine 22: 1079-1087, 2015 .

19. Cheng R, Chen J, Wang Y, Ge Y, Huang Z and Zhang G: Dihydrotanshinone induces apoptosis of SGC7901 and MGC803 cells via activation of JNK and p38 signalling pathways. Pharm Biol 54: 3019-3025, 2016.

20. Cheng J, Fan YQ, Liu BH, Zhou H, Wang JM and Chen QX ACSL4 suppresses glioma cells proliferation via activating ferroptosis. Oncol Rep 43: 147-158, 2020.

21. Houessinon A, Francois C, Sauzay C, Louandre C, Mongelard G, Godin C, Bodeau S, Takahashi S, Saidak Z, Gutierrez L, et al: Metallothionein-1 as a biomarker of altered redox metabolism in hepatocellular carcinoma cells exposed to sorafenib. Mol Cancer 15: 38, 2016.

22. Ooko E, Saeed ME, Kadioglu O, Sarvi S, Colak M, Elmasaoudi K, Janah R, Greten HJ and Efferth T: Artemisinin derivatives induce iron-dependent cell death (ferroptosis) in tumor cells. Phytomedicine 22: 1045-1054, 2015.

23. Dixon SJ, Lemberg KM, Lamprecht MR, Skouta R, Zaitsev EM, Gleason CE, Patel DN, Bauer AJ, Cantley AM, Yang WS, et al: Ferroptosis: An iron-dependent form of nonapoptotic cell death Cell 149: 1060-1072, 2012.

24. Xie Y, Hou W, Song X, Yu Y, Huang J, Sun X, Kang R and Tang D: Ferroptosis: Process and function. Cell Death Differ 23: 369-379, 2016.

25. Dixon SJ and Stockwell BR: The role of iron and reactive oxygen species in cell death. Nat Chem Biol 10: 9-17, 2014.

26. Stockwell BR, Friedmann Angeli JP, Bayir H, Bush AI, Conrad M, Dixon SJ, Fulda S, Gascón S, Hatzios SK, Kagan VE, et al: Ferroptosis: A regulated cell death nexus linking metabolism, redox biology, and disease. Cell 171: 273-285, 2017

27. Lu B, Chen XB, Ying MD, He QJ, Cao J and Yang B: The role of ferroptosis in cancer development and treatment response. Front Pharmacol 8: 992, 2018

28. Wenzel SE, Tyurina YY, Zhao J, St Croix CM, Dar HH, Mao G, Tyurin VA, Anthonymuthu TS, Kapralov AA, Amoscato AA, et al: PEBP1 wardens ferroptosis by enabling lipoxygenase generation of lipid death signals. Cell 171: 628-641, 2017.

29. Doll S, Proneth B, Tyurina YY, Panzilius E, Kobayashi S, Ingold I, Irmler M, Beckers J, Aichler M, Walch A, et al: ACSL4 dictates ferroptosis sensitivity by shaping cellular lipid composition. Nat Chem Biol 13: 91-98, 2017.

30. Sun X, Ou Z, Chen R, Niu X, Chen D, Kang R and Tang D: Activation of the p62-Keap1-NRF2 pathway protects against ferroptosis in hepatocellular carcinoma cells. Hepatology 63: 173-184, 2016.

31. Lin YS, Shen YC, Wu CY, Tsai YY, Yang YH, Lin YY, Kuan FC, Lu CN, Chang GH, Tsai MS, et al: Danshen improves survival of patients with breast cancer and dihydroisotanshinone I induces ferroptosis and apoptosis of breast cancer cells. Front Pharmacol 10: 1226, 2019.

32. Parhamifar L, Andersen H and Moghimi SM: Lactate dehydrogenase assay for assessment of polycation cytotoxicity. Methods Mol Biol 1943: 291-299, 2019.

33. Shan Y, Liu B, Li L, Chang N, Li L, Wang H, Wang D, Feng H, Cheung C, Liao M, et al: Regulation of PINK1 by NR2B-containing NMDA receptors in ischemic neuronal injury. J Neurochem 111: 1149-1160, 2009.

34. Baysal M, Ilgin S, Kilic G, Kilic V, Ucarcan S and Atli O: Reproductive toxicity after levetiracetam administration in male rats: Evidence for role of hormonal status and oxidative stress PLoS One 12: e175990, 2017.

35. Imai H, Matsuoka M, Kumagai T, Sakamoto T and Koumura T: Lipid peroxidation-dependent cell death regulated by GPx4 and ferroptosis. Curr Top Microbiol Immunol 403: 143-170, 2017.

36. Li C, Deng X, Zhang W, Xie X, Conrad M, Liu Y, Angeli JPF and Lai L: Novel allosteric activators for ferroptosis regulator glutathione peroxidase 4. J Med Chem 62: 266-275, 2019.

37. Yuan H, Li X, Zhang X, Kang R and Tang D: Identification of ACSL4 as a biomarker and contributor of ferroptosis. Biochem Biophys Res Commun 478: 1338-1343, 2016. 
38. Yang WS and Stockwell BR: Ferroptosis: Death by lipid peroxidation. Trends Cell Biol 26: 165-176, 2016.

39. Zhou Y: The protective effects of cryptochlorogenic acid on $\beta$-cells function in diabetes in vivo and vitro via inhibition of ferroptosis. Diabetes Metab Syndr Obes 13: 1921-1931, 2020.

40. Friedmann Angeli AJ, Schneider M, Proneth B, Tyurina YY, Tyurin VA, Hammond VJ, Herbach N, Aichler M, Walch A, Eggenhofer E, et al: Inactivation of the ferroptosis regulator Gpx4 triggers acute renal failure in mice. Nat Cell Biol 16: 1180-1191, 2014.

41. Zilka O, Shah R, Li B, Friedmann Angeli JP, Griesser M, Conrad M and Pratt DA: On the mechanism of cytoprotection by ferrostatin-1 and liproxstatin-1 and the role of lipid peroxidation in ferroptotic cell death. ACS Cent Sci 3: 232-243, 2017.

42. Wang L, Ma R, Liu C, Liu H, Zhu R, Guo S, Tang M, Li Y, Niu J, Fu M, et al: Salvia miltiorrhiza: A potential red light to the development of cardiovascular diseases. Curr Pharm Des 23 1077-1097, 2017.

43. Xu Y, Chen T, Li X, Qu YK, An JN, Zheng HX, Zhang ZJ and Lin N: Salvia miltiorrhiza bunge increases estrogen level without side effects on reproductive tissues in immature/ovariectomized mice. Aging (Albany NY) 9: 156-172, 2016.

44. Zhang PR and Lü Q: A study on anticancer activity of tanshinone II A against human breast cancer. Sichuan Da Xue Xue Bao Yi Xue Ban 40: 245-249, 2009 (In Chinese).

45. Zhang HS, Zhang FJ, Li H, Liu Y, Du GY and Huang YH: Tanshinone A inhibits human esophageal cancer cell growth through miR-122-mediated PKM2 down-regulation. Arch Biochem Biophys 598: 50-56, 2016.

46. Liu D, Yuan R, Yang F and Zhang D: Effects of tanshinones mediated by forkhead box O3a transcription factor on the proliferation and apoptosis of lung cancer cells. Oncol Lett 17: 450-455, 2019.

47. Mosaddik MA: In vitro cytotoxicity of tanshinones isolated from Salvia miltiorrhiza Bunge against P388 lymphocytic leukemia cells. Phytomedicine 10: 682-685, 2003.

48. Park JW, Lee SH, Yang MK, Lee JJ, Song MJ, Ryu SY, Chung HJ, Won HS, Lee CS, Kwon SH, et al: 15,16-dihydrotanshinone I, a major component from Salvia miltiorrhiza Bunge (Dansham), inhibits rabbit platelet aggregation by suppressing intracellular calcium mobilization. Arch Pharm Res 31: 47-53, 2008.

49. Suk FM, Jou WJ, Lin RJ, Lin SY, Tzeng FY and Liang YC: 15,16-Dihydrotanshinone I-induced apoptosis in human colorectal cancer cells: Involvement of ATF3. Anticancer Res 33 : 3225-3231, 2013

50. Wu CY, Yang YH, Lin YY, Kuan FC, Lin YS, Lin WY, Tsai MY, Yang JJ, Cheng YC, Shu LH, et al: Anti-cancer effect of danshen and dihydroisotanshinone I on prostate cancer: Targeting the crosstalk between macrophages and cancer cells via inhibition of the STAT3/CCL2 signaling pathway. Oncotarget 8 : 40246-40263, 2017

51. Lin YY, Lee IY, Huang WS, Lin YS, Kuan FC, Shu LH, Cheng YC, Yang YH and Wu CY: Corrigendum to 'Danshen improves survival of patients with colon cancer and dihydroisotanshinone I inhibit the proliferation of colon cancer cells via apoptosis and skp2 signaling pathway' J Ethnopharmacol 213: 446, 2018.

52. Ju LX, Chen Z and Ren RZ: Progress in research on the treatment of primary liver cancer with traditional Chinese medicine for activating blood to resolve stasis. Zhong Xi Yi Jie He Xue Bao 3 : 491-494, 2005 (In Chinese).

53. Aquilanti E, Miller J, Santagata S, Cahill DP and Brastianos PK: Updates in prognostic markers for gliomas. Neuro Oncol 20 (Suppl 7): vii17-vii26, 2018

54. Mair DB, Ames HM and Li R: Mechanisms of invasion and motility of high-grade gliomas in the brain. Mol Biol Cell 29: 2509-2515, 2018

55. Hanaei S, Afshari K, Hirbod-Mobarakeh A, Mohajer B, Amir DD and Rezaei N: Therapeutic efficacy of specific immunotherapy for glioma: A systematic review and meta-analysis. Rev Neurosci 29: 443-461, 2018.
56. Natsume A and Yoshida J: Gene therapy for high-grade glioma: Current approaches and future directions. Cell Adh Migr 2: 186-191, 2008.

57. Latunde-Dada GO: Ferroptosis: Role of lipid peroxidation, iron and ferritinophagy. Biochim Biophys Acta Gen Subj 1861: 1893-1900, 2017.

58. Zhang L, Liu W, Liu F, Wang Q, Song M, Yu Q, Tang K, Teng T, Wu D, Wang $\mathrm{X}$, et al: IMCA Induces ferroptosis mediated by SLC7A11 through the AMPK/mTOR pathway in colorectal cancer. Oxid Med Cell Longev 2020: 1675613, 2020.

59. Homma T and Fujii J: Application of glutathione as anti-oxidative and anti-aging drugs. Curr Drug Metab 16: 560-571, 2015

60. Wang Y, Zhao Y, Wang H, Zhang C, Wang M, Yang Y, Xu X and $\mathrm{Hu} \mathrm{Z}$ : Histone demethylase KDM3B protects against ferroptosis by upregulating SLC7A11. FEBS Open Bio 10: 637-643, 2020.

61. Kim DH, Kim WD, Kim SK, Moon DH and Lee SJ: TGF- $\beta 1$-mediated repression of SLC7A11 drives vulnerability to GPX4 inhibition in hepatocellular carcinoma cells. Cell Death Dis 11: 406, 2020

62. Friedmann AJ and Conrad M: Selenium and GPX4, a vital symbiosis. Free Radic Biol Med 127: 153-159, 2018.

63. Ingold I, Berndt C, Schmitt S, Doll S, Poschmann G, Buday K, Roveri A, Peng X, Freitas FP, Seibt T, et al: Selenium utilization by GPX4 is required to prevent hydroperoxide-induced ferroptosis. Cell 172: 409-422.e21, 2018

64. Sui X, Zhang R, Liu S, Duan T, Zhai L, Zhang M, Han X, Xiang Y, Huang X, Lin H and Xie T: RSL3 drives ferroptosis through GPX4 Inactivation and ROS production in colorectal cancer. Front Pharmacol 9: 1371, 2018

65. Belavgeni A, Bornstein SR and Linkermann A: Prominin-2 suppresses ferroptosis sensitivity. Dev Cell 51: 548-549, 2019.

66. Li W, Li W, Leng Y, Xiong Y and Xia Z: Ferroptosis is involved in diabetes myocardial ischemia/reperfusion injury through endoplasmic reticulum stress. DNA Cell Biol 39: 210-225, 2019.

67. Liu N, Lin $X$ and Huang C: Activation of the reverse transsulfuration pathway through NRF2/CBS confers erastin-induced ferroptosis resistance. Br J Cancer 122: 279-292, 2019.

68. Guo J, Xu B, Han Q, Zhou H, Xia Y, Gong C, Dai X, Li Z and Wu G: Ferroptosis: A novel anti-tumor action for cisplatin. Cancer Res Treat 50: 445-460, 2018.

69. Louandre C, Marcq I, Bouhlal H, Lachaier E, Godin C, Saidak Z, François C, Chatelain D, Debuysscher V, Barbare JC, et al: The retinoblastoma $(\mathrm{Rb})$ protein regulates ferroptosis induced by sorafenib in human hepatocellular carcinoma cells. Cancer Lett 356 (2 Pt B): 971-977, 2015

70. Louandre C, Ezzoukhry Z, Godin C, Barbare JC, Mazière JC, Chauffert B and Galmiche A: Iron-dependent cell death of hepatocellular carcinoma cells exposed to sorafenib. Int J Cancer 133: 1732-1742, 2013

71. Yu H, Yang C, Jian L, Guo S, Chen R, Li K, Qu F, Tao K, Fu Y, Luo F and Liu S: Sulfasalazineinduced ferroptosis in breast cancer cells is reduced by the inhibitory effect of estrogen receptor on the transferrin receptor. Oncol Rep 42: 826-838, 2019.

72. Bach DH, Luu TT, Kim D, An YJ, Park S, Park HJ and Lee SK BMP4 upregulation is associated with acquired drug resistance and fatty acid metabolism in EGFR-mutant non-small-cell lung cancer cells. Mol Ther Nucleic Acids 12: 817-828, 2018.

73. Zhang C, Liao Y, Liu P, Du Q, Liang Y, Ooi S, Qin S, He S, Yao S and Wang W: FABP5 promotes lymph node metastasis in cervical cancer by reprogramming fatty acid metabolism. Theranostics 10: 6561-6580, 2020.

74. Radif Y, Ndiaye H, Kalantzi V, Jacobs R, Hall A, Minogue S and Waugh MG: The endogenous subcellular localisations of the long chain fatty acid-activating enzymes ACSL3 and ACSL4 in sarcoma and breast cancer cells. Mol Cell Biochem 448: 275-286, 2018.

This work is licensed under a Creative Commons

Attribution-NonCommercial-NoDerivatives 4.0 International (CC BY-NC-ND 4.0) License. 